\title{
Some Fixed Point Theorems under Weak Semicompatibility
}

\author{
A. S. Saluja and Mukesh Kumar Jain \\ Government JH Post Graduate College, Betul, Madhya Pradesh 460001, India \\ Correspondence should be addressed to Mukesh Kumar Jain; mukesh.jain2007@rediffmail.com
}

Received 4 November 2013; Accepted 24 December 2013; Published 27 April 2014

Academic Editors: Z. Guo and P. K. Sahoo

Copyright (C) 2014 A. S. Saluja and M. K. Jain. This is an open access article distributed under the Creative Commons Attribution License, which permits unrestricted use, distribution, and reproduction in any medium, provided the original work is properly cited.

The aim of the present paper is to prove some fixed point theorems by using the recent notion "weak semicompatibility." The new notion is proper generalization of semicompatibility and can be applicable on commuting and compatible maps. We used compatible and absorbing mappings to prove theorems which also include (E.A.) property.

\section{Introduction and New Definitions}

In 1995, Cho et al. [1] introduced the concept of semicompatibility and obtained the first result that established a situation in which a collection of mappings has a fixed point. They defined a pair of self-maps $(S, T)$ to be semicompatible if

(a) $S x=T x \Rightarrow S T x=T S x$ and

(b) $\lim _{n \rightarrow \infty} S x_{n}=\lim _{n \rightarrow \infty} T x_{n}=x$. For some $x \in X$, implying $\lim _{n \rightarrow \infty} S T x_{n}=T x$ holds.

Singh and Jain [2] observe that (b) implies (a). Hence they defined the semicompatibility by condition (b) only.

Let $(X, d)$ be a metric space and let $f$ and $g$ be two maps from $(X, d)$ into itself. $f$ and $g$ are commuting maps if $f g x=$ $g f x$ for all $x$ in $X$.

To generalize the notion of commuting maps, Sessa [3] introduced the concept of weakly commuting maps. He defines $f$ and $g$ as being weakly commuting if $d(f g x, g f x) \leq$ $d(f x, g x)$ for all $x \in X$. Obviously, commuting maps are weakly commuting but the converse is not true.

In 1986, Jungck [4] gave more generalized commuting and weakly commuting maps called compatible maps. $f$ and $g$ are called compatible if

$$
\lim _{n \rightarrow \infty} d\left(f g x_{n}, g f x_{n}\right)=0 .
$$

Whenever $\left\{x_{n}\right\}$ is a sequence in $X$ such that $\lim _{n \rightarrow \infty} f x_{n}=$ $\lim _{n \rightarrow \infty} g x_{n}=t$ for some $t \in X$, clearly, weakly commuting maps are compatible, but the implication is not reversible (see [4]).
Afterwards, Jungck et al. [5] made another generalization of weakly commuting maps by introducing the concept of compatible maps of type $(A)$. Previous $f$ and $g$ are said to be compatible of type $(A)$ if in place of (1) we have the two following conditions:

$$
\begin{aligned}
& \lim _{n \rightarrow \infty} d\left(f g x_{n}, g g x_{n}\right)=0, \\
& \lim _{n \rightarrow \infty} d\left(g f x_{n}, f f x_{n}\right)=0 .
\end{aligned}
$$

It is clear to see that weakly commuting maps are compatible of type $(A)$; from [5] it follows that the implication is not reversible.

Two self-maps $f$ and $g$ of metric space $(X, d)$ are called $g$-compatible ([6] cited from [7]) if $\lim d\left(g f x_{n}, f f x_{n}\right)=$ 0 , whenever $\left\{x_{n}\right\}$ is a sequence in $X$ such that $\lim f x_{n}=$ $\lim g x_{n}=t$ for some $t$ in $X$. Similarly, two self-maps $f$ and $g$ of metric space $(X, d)$ are called $f$-compatible ([6] cited from [7]) if $\lim d\left(f g x_{n} g g x_{n}\right)=0$, whenever $\left\{x_{n}\right\}$ is a sequence in $X$ such that $\lim f x_{n}=\lim g x_{n}=t$ for some $t$ in $X$.

Two self-mappings $f$ and $g$ of a metric space $(X, d)$ are called $R$ weakly commuting [8] at a point $x$ in $X$ if $d(f g x, g f x) \leq R d(f x, g x)$ for some $R>0$.

The two self-maps $f$ and $g$ of a metric space $(X, d)$ are called $R$ weakly commuting of type $\left(A_{g}\right)$ [9] if there exists some positive real number $R$ such that $d(f f x, g f x) \leq$ $R d(f x, g x)$ for all $x$ in $X$.

The two self-maps $f$ and $g$ of a metric space $(X, d)$ are called $R$ weakly commuting of type $\left(A_{f}\right)$ [9] if there exists some positive real number $R$ such that $d(f g x, g g x) \leq$ 
$R d(f x, g x)$ for all $x$ in $X$. It may be noted that compatible mappings $f$ and $g$ can be $R$ weakly commuting of types $\left(A_{f}\right)$ and $\left(A_{g}\right)$.

Let $f$ and $g(f \neq g)$ be two self-maps of metric space $(X, d)$; then $f$ will be called $g$-absorbing [10] if there exists a real number $R>0$ such that $d(g x, g f x) \leq R d(f x, g x)$ for all $x \in X$. Similarly, let $f$ and $g(f \neq g)$ be two self-maps of metric space $(X, d)$; then $g$ will be called $f$-absorbing [10] if there exists a real number $R>0$ such that $d(f x, f g x) \leq$ $R d(f x, g x)$ for all $x \in X$.

Let $f$ and $g$ be two self-mappings of metric space $(X, d)$. The maps $f$ and $g$ satisfy the E.A. property [11] if there exists a sequence $\left\{x_{n}\right\}$ in $X$ such that $\lim f x_{n}=\lim g x_{n}=t$ for some $t \in X$.

Two self-maps $f$ and $g$ of metric space $(X, d)$ are said to be $f$-compatible of type $(E)[12]$ if $\lim f f x_{n}=\lim f g x_{n}=$ $g t$, whenever $\left\{x_{n}\right\}$ is a sequence in $X$ such that $\lim f x_{n}=$ $\lim g x_{n}=t$ for some $t$ in $X$. Similarly, two self-maps $f$ and $g$ of metric space $(X, d)$ are said to be $g$-compatible of type $(E)$ [12] if $\lim g g x_{n}=\lim g f x_{n}=f t$, whenever $\left\{x_{n}\right\}$ is a sequence in $X$ such that $\lim f x_{n}=\lim g x_{n}=t$ for some $t$ in $X$.

Pant et al. [13] introduced a notion of weak reciprocal continuity as follows.

Definition 1. Two self-mappings $f$ and $g$ of metric space $(X, d)$ will be called weakly reciprocally continuous if $\lim f g x_{n}=f t$ or $\lim g f x_{n}=g t$, whenever $\left\{x_{n}\right\}$ is a sequence in $X$ such that $\lim f x_{n}=\lim g x_{n}=t$ for some $t$ in $X$.

Further Saluja et al. [14] introduced a notion of weak semicompatibility as follows.

Definition 2. Two self-mappings $f$ and $g$ of a metric space $(X, d)$ will be called weak semicompatible mappings if $\lim _{n \rightarrow \infty} f g x_{n}=g t$ or $\lim _{n \rightarrow \infty} g f x_{n}=f t$, whenever $\left\{x_{n}\right\}$ is a sequence in $X$ such that $\lim _{n \rightarrow \infty} f x_{n}=\lim _{n \rightarrow \infty} g x_{n}=t$ for some $t$ in $X$.

Example 3 (see [14]). Let $X=[0,1]$ and $d$ be the usual metric on $X$. Define $f, g: X \rightarrow X$ by

$$
\begin{aligned}
& f x=x \quad \forall x \in[0,1], \\
& g x= \begin{cases}0, & x \in(0,1], \\
1, & x=0 .\end{cases}
\end{aligned}
$$

Taking $\left\{x_{n}\right\}=1 / n$, since $\lim f x_{n}=\lim g x_{n}=0$, we have

$$
\begin{gathered}
\lim f g x_{n}=\lim f(0)=0 \neq g(0), \\
\lim g f x_{n}=\lim g\left(x_{n}\right)=\lim g\left(\frac{1}{n}\right)=0=f(0) .
\end{gathered}
$$

Therefore $f$ and $g$ are weak semicompatible.

Also here maps $f$ and $g$ have no common fixed point.

Now we give some more definitions to improve this result.

Definition 4 (see [15]). Let $X$ be a set and $f$ and $g$ be self-maps of $X$. A point $x$ in $X$ is called coincidence point of $f$ and $g$ if and only if $f x=g x$. One will call $w=f x=g x$ a point of coincidence of $f$ and $g$.
Definition 5 (see [16]). Two self-maps $f$ and $g$ of a set $X$ are occasionally weakly compatible (owc) if and only if there is a point $x$ in $X$ which is coincidence point of $f$ and $g$ at which $f$ and $g$ commute.

Lemma 6 (see [15]). Let $X$ be a set and let $f$ and $g$ be owc self-maps on $X$. If $f$ and $g$ have a unique point of coincidence, $w:=f x=g x$, then $w$ is the unique common fixed point of $f$ and $g$.

Definition 7 (see [17]). Two self-mappings $f$ and $g$ on a metric space $(X, d)$ are called conditionally commuting if they commute on nonempty subset of the set of coincidence points whenever the set of their coincidence points is nonempty.

From the definition itself, it is clear that if two maps are weakly compatible or owc, then they are necessarily conditionally commuting; however, the conditionally commuting mappings are not necessarily weakly commuting or owc.

Definition 8. Let $X$ be a set. A symmetric on $X$ is a mapping $d: X \times X \rightarrow[0, \infty)$ such that

$$
\begin{gathered}
d(x, y)=0 \quad \text { iff } x=y, \\
d(x, y)=d(y, x) \quad \text { for } x, y \in X .
\end{gathered}
$$

Theorem 9. Let $X$ be a set with a symmetric d. Suppose that $f$ and $g$ are owc self-maps of $X$ satisfying

(a) $d(f x, f y) \geq a d(g x, g y)+b d(g x, f x)+c d(g y, f y)$,

where $a, c>1, b \in R$, such that $a+b+c>1$. Then $f$ and $g$ have a unique common fixed point.

Proof. Since the maps are owc, there exists a point $x$ such that $f x=g x$ and $f g x=g f x$. Substituting $(a)$ with $y=g x$ gives

$$
\begin{aligned}
d(f x, f g x) \geq & a d(g x, g f x)+b d(g x, f x) \\
& +c d(g f x, f g x)=a d(g x, f g x) .
\end{aligned}
$$

Thus, $0 \geq(a-1) d(f x, f g x)$, which implies that $f x$ is a fixed point of $f$. But, since $g f x=f g x, f x$ is also a fixed point of $g$.

Suppose that $p$ and $q$ are common fixed point of $f$ and $g$. Substituting (1) with

$$
\begin{aligned}
d(p, q)=d(f p, f q) \geq & a d(g p, g q)+b d(g p, f p) \\
& +c d(g q, f q)=a d(p, q),
\end{aligned}
$$

implies that $p=q$

Lemma 10 (see [18]). If $f$ and $g$ are compatible of type $(A)$, then they are owc, but the converse is not true in general.

Now we give the following lemma with the fact of [18].

Lemma 11. If $f$ and $g$ are either $f$-compatible or $g$-compatible, then they are owc, but the converse is not true in general. We give the following examples to prove this. 
Example 12. Let $X=[0, \infty)$ with the usual metric. Define $f, g: X \rightarrow X$ by

$$
\begin{gathered}
f x= \begin{cases}3, & \text { if } x \in[0,1), \\
x, & \text { if } x \in[1, \infty),\end{cases} \\
g x= \begin{cases}2, & \text { if } x \in[0,1), \\
\frac{1}{x}, & \text { if } x \in[1, \infty) .\end{cases}
\end{gathered}
$$

We have $f(1)=g(1)=1$ and $f g(1)=g f(1)=1$; that is, $f$ and $g$ are owc. Now consider $x_{n}=1+1 / n$ for $n \in\{1,2,3 \ldots\}$; we have $\lim f x_{n}=\lim f(1+1 / n)=1$ and $\lim g x_{n}=\lim g(1+$ $1 / n)=1$. But $\lim f g x_{n}=\lim f g(1+1 / n)=3$ and $\lim g g x_{n}=$ $\lim g g(1+1 / n)=2$; therefore $\lim d\left(f g x_{n}, g g x_{n}\right) \neq 0$; hence $f$ and $g$ are not $g$-compatible.

Example 13. Let $X=[1,5]$ with the usual metric. Define $f, g$ : $X \rightarrow X$ by

$$
\begin{gathered}
f x= \begin{cases}1, & \text { if } x=1, \\
5, & \text { if } x \in(1,5],\end{cases} \\
g x= \begin{cases}1, & \text { if } x=1, \\
x+4, & \text { if } x \in(1,5] .\end{cases}
\end{gathered}
$$

We have $f(1)=g(1)=1$ and $f g(1)=g f(1)=1$; that is, $f$ and $g$ are owc. Now consider $x_{n}=1+1 / n$ for $n \in\{1,2,3 \ldots\}$; we have $\lim f x_{n}=\lim f(1+1 / n)=5$ and $\lim g x_{n}=$ $\lim g(1+1 / n)=5$. But $\lim g f x_{n}=\lim g f(1+1 / n)=g(5)=9$ and $\lim f f x_{n}=\lim f f(1+1 / n)=\lim f(5)=5$; therefore $\lim d\left(g f x_{n}, f f x_{n}\right) \neq 0$; hence $f$ and $g$ are not $f$-compatible.

Lemma 14. If $f$ and $g$ are $f$-compatible of type (E) or $g$ compatible of type (E), then they are owc, but the converse is not true in general. Here we prove the converse condition.

Proof. If $X=[1, \infty)$ with the usual metric, define $f$ and $g$ by

$$
\begin{aligned}
& f x= \begin{cases}\frac{1}{x}, & x \in[1,2), \\
1+x, & x \in[2, \infty),\end{cases} \\
& g x= \begin{cases}\frac{1}{x^{2}}, & x \in[1,2), \\
5-x, & x \in[2, \infty) .\end{cases}
\end{aligned}
$$

We have $f(1)=g(1), f(2)=g(2)$, and also $f g(1)=g f(1)$, but $f g(2) \neq g f(2)$. This implies that $f$ and $g$ are owc.

If we take sequence $x_{n}=2+1 / n$, then

$$
\lim f x_{n}=\lim g x_{n}=3,
$$

$\lim f f x_{n}=\lim f f\left(2+\frac{1}{n}\right)=\lim f\left(3+\frac{1}{n}\right)=4 \neq g(3)$,

$$
\lim f g x_{n}=\lim f g\left(2+\frac{1}{n}\right)=\lim f\left(3-\frac{1}{n}\right)=4 \neq g(3) \text {. }
$$

This implies that $f$ and $g$ are not $f$-compatible of type (E).

\section{Main Results}

Theorem 15. Let $f$ and $g$ be weak semicompatible, $R$ weakly commuting type of $A_{f}$, and self-mappings of a complete metric space $(X, d)$ such that

(a) $f(X) \subseteq g(X)$;

(b) $d(f x, f y) \geq a d(g x, g y)+b d(f x, g x)+c d(f y, g y)$; $a, c>1(a+c>0)$ and $b \in R$ such that $a+b+c>1$;

(c) $f$ and $g$ are either $f$-compatible of type (E) or $g$ compatible of type $(E)$.

If fand $g$ are conditional commuting, then $f$ and $g$ have a common fixed point in $X$.

Proof. Let $x_{0}$ be any point in $X$. Since $f(X) \subseteq g(X)$, there exist $x_{1} \in X$ such that $f x_{1}=g x_{0}=y_{0}$. Similarly, we can have a sequence

$$
f x_{n+1}=g x_{n}=y_{n}
$$

Now by (b), we have

$$
\begin{aligned}
d\left(f x_{n}, f x_{n+1}\right) \geq & a d\left(g x_{n}, g x_{n+1}\right)+b d\left(f x_{n}, g x_{n}\right) \\
& +c d\left(f x_{n+1}, g x_{n+1}\right), \\
d\left(y_{n-1}, y_{n}\right) \geq & a d\left(y_{n}, y_{n+1}\right)+b d\left(y_{n-1}, y_{n}\right) \\
& +c d\left(y_{n}, y_{n+1}\right), \\
d\left(y_{n}, y_{n+1}\right) \leq & \frac{1-b}{a+c} d\left(y_{n-1}, y_{n}\right) .
\end{aligned}
$$

Since $(1-b) /(a+c)=k<1(a+b+c>1)$,

$$
d\left(y_{n}, y_{n+1}\right) \leq k d\left(y_{n-1}, y_{n}\right)
$$

Similarly, we can obtain $d\left(y_{n-1}, y_{n}\right) \leq k d\left(y_{n-2}, y_{n-1}\right)$. Therefore by (15) we have $d\left(y_{n}, y_{n+1}\right) \leq k^{2} d\left(y_{n-2}, y_{n-1}\right)$. By the same way, we can have

$$
d\left(y_{n}, y_{n+1}\right) \leq k^{n} d\left(y_{0}, y_{1}\right) \text {. }
$$

Now we will show that $\left\{y_{n}\right\}$ is a Cauchy sequence. For any integer $p>0$, we get

$$
\begin{gathered}
d\left(y_{n}, y_{n+p}\right) \leq d\left(y_{n}, y_{n+1}\right)+d\left(y_{n+1}, y_{n+2}\right) \\
+\cdots+d\left(y_{n+p-1}, y_{n+p}\right), \text { now by }(16) \\
d\left(y_{n}, y_{n+p}\right) \leq k^{n} d\left(y_{0}, y_{1}\right)+k^{n+1} d\left(y_{0}, y_{1}\right) \\
+\cdots+k^{n+p-1} d\left(y_{0}, y_{1}\right), \\
d\left(y_{n}, y_{n+p}\right) \leq k^{n}\left[1+k+k^{2}+k^{3}+\cdots+k^{p-1}\right] d\left(y_{0}, y_{1}\right), \\
d\left(y_{n}, y_{n+p}\right) \leq \frac{k^{n}}{1-k} d\left(y_{0}, y_{1}\right) .
\end{gathered}
$$

Since $k<1$, then taking limit $n \rightarrow \infty$ we have $d\left(y_{n}, y_{n+p}\right) \rightarrow$ 0 . Therefore $\left\{y_{n}\right\}$ is a Cauchy sequence. Since $X$ is complete, there exists a point $t$ in $X$ such that $\left\{y_{n}\right\} \rightarrow t$ as $n \rightarrow \infty$; moreover $y_{n}=f x_{n+1}=g x_{n} \rightarrow t$ for $t \in X$. 
Case 1 ( $f$ and $g$ are $f$-compatible of type $(E)$ ). Since $f$ and $g$ are weak semicompatible, this yields either $\lim f g x_{n}=g t$ or $\lim g f x_{n}=f t$. First we take $\lim g f x_{n}=f t$.

Since $f$ and $g$ are $f$-compatible of type $(E)$, this yields $\lim f f x_{n}=\lim f g x_{n}=g t$. Now by (b), we have

$$
\begin{aligned}
d\left(f f x_{n}, f t\right) \geq & a d\left(g f x_{n}, g t\right)+b d\left(f f x_{n}, g f x_{n}\right) \\
& +c d(f t, g t),
\end{aligned}
$$

Now limiting $n \rightarrow \infty$ yields

$$
d(g t, f t) \geq a d(f t, g t)+b d(g t, f t)+c d(f t, g t),
$$

Since $a+b+c>1$, this yields $f t=g t$.

Now the conditional commutativity of $f$ and $g$ implies $f g t=g f t$ or $f g t=g f t=f f t=g g t$. Now by (b), we have

$$
\begin{aligned}
& d(f f t, f t) \geq a d(g f t, g t)+b d(f f t, g f t)+c d(f t, g t), \\
& d(f f t, f t) \geq a d(f f t, f t) .
\end{aligned}
$$

Since $a>1$, this implies $f f t=f t$ or $f f t=g f t=f t$. Hence $f t$ is common fixed point of $f$ and $g$.

Now we take $\lim f g x_{n}=g t$.

Since $f$ and $g$ are $f$-compatible of type $(E)$, this yields $\lim f f x_{n}=\lim f g x_{n}=g t$.

Also $f$ and $g$ are $R$-weakly commuting type of $A_{f}$ and this implies $d\left(f g x_{n}, g g x_{n}\right) \leq R d\left(f x_{n}, g x_{n}\right)$.

Now limiting $n \rightarrow \infty$ yields $\lim g g x_{n}=g t$. Again by (b), we have

$$
\begin{aligned}
d\left(f x_{n}, f g x_{n}\right) \geq & a d\left(g x_{n}, g g x_{n}\right)+b d\left(f x_{n}, g x_{n}\right) \\
& +c d\left(f g x_{n}, g g x_{n}\right) .
\end{aligned}
$$

Now limiting $n \rightarrow \infty$ yields

$$
d(t, g t) \geq a d(t, g t)+b d(t, t)+c d(g t, g t) .
$$

Since $a>1$ implies $g t=t$, now by $(b)$, we get

$$
\begin{aligned}
d\left(f x_{n}, f t\right) \geq & a d\left(g x_{n}, g t\right)+b d\left(f x_{n}, g x_{n}\right) \\
& +c d(f t, g t) .
\end{aligned}
$$

Now limiting $n \rightarrow \infty$ with $c>1$ yields $f t=t$, and hence $f t=g t=t$ and $t$ is common fixed point of $f$ and $g$.

Case 2 ( $f$ and $g$ are $g$-compatible of type $(E)$ ). Since $f$ and $g$ are weak semicompatible, this yields either $\lim f g x_{n}=g t$ or $\lim g f x_{n}=f t$.

First we take $\lim g f x_{n}=f t$; since $f$ and $g$ are $g$ compatible of type $(E)$, this yields $\lim g g x_{n}=\lim g f x_{n}=f t$. Also $f$ and $g$ are $R$-weakly commuting type of $A_{f}$ and this implies $d\left(f g x_{n}, g g x_{n}\right) \leq R d\left(f x_{n}, g x_{n}\right)$. Now limiting $n \rightarrow$ $\infty$, we get $\lim f g x_{n}=f t$. Now by (b), we get

$$
\begin{aligned}
d\left(f g x_{n}, f t\right) \geq & a d\left(g g x_{n}, g t\right)+b d\left(f g x_{n}, g g x_{n}\right) \\
& +c d(f t, g t) .
\end{aligned}
$$

Now limiting $n \rightarrow \infty$, we get $d(f t, f t) \geq a d(f t, g t)+$ $b d(f t, f t)+c d(f t, g t)$, Since $a+c>0$, this yields $f t=g t$.

Now the conditional commutativity of $f$ and $g$ implies $f g t=g f t$ or $f g t=g f t=f f t=g g t$. Now by (b), we get

$$
\begin{aligned}
& d(f f t, f t) \geq a d(g f t, g t)+b d(f f t, g f t)+c d(f t, g t), \\
& d(f f t, f t) \geq a d(f f t, f t) .
\end{aligned}
$$

Since $a>1$, this implies $f f t=f t$ or $f f t=g f t=f t$. Hence $f t$ is common fixed point of $f$ and $g$.

Now we take $\lim f g x_{n}=g t$.

Since $f$ and $g$ are $g$-compatible of type $(E)$, this yields $\lim g g x_{n}=\lim g f x_{n}=f t$. Now by (b) $d\left(f g x_{n}, f t\right) \geq$ $a d\left(g g x_{n}, g t\right)+b d\left(f g x_{n}, g g x_{n}\right)+c d(f t, g t)$. Limiting $n \rightarrow \infty$ yields $d(g t, f t) \geq a d(f t, g t)+b d(g t, f t)+c d(f t, g t)$, since $a+b+c>1$ yields $f t=g t$.

Now the conditional commutativity of $f$ and $g$ implies $f g t=g f t$ or $f g t=g f t=f f t=g g t$. Now by (b), we get

$$
\begin{aligned}
& d(f f t, f t) \geq a d(g f t, g t)+b d(f f t, g f t)+c d(f t, g t), \\
& d(f f t, f t) \geq a d(f f t, f t) .
\end{aligned}
$$

Since $a>1$, this implies $f f t=f t$ or $f f t=g f t=f t$. Hence $f t$ is common fixed point of $f$ and $g$.

Example 16. Let $x, y \in X(x \geq y)$ and $X=[1,7]$ and $d$ be the usual metric on $X$. Define $f, g: X \rightarrow X$ as follows:

$$
\begin{array}{rr}
f x=1 \quad \text { if } x=1, \quad f x=\frac{x+5}{2} \quad \text { if } x>5, \\
f x=7 \quad \text { if } 1<x \leq 5, \\
g x=1 \quad \text { if } x=1, \quad g x=x \quad \text { if } x \geq 5, \\
g x=6 \quad \text { if } 1<x<5 .
\end{array}
$$

When we take constant sequence $\left\{x_{n}\right\}=1$, then $\lim f x_{n}=$ $\lim g x_{n}=1, \lim f g x_{n}=1=g(1)$, and $\lim g f x_{n}=1=f(1)$, but if we take sequence $x_{n}=5+1 / n, \lim f x_{n}=\lim g x_{n}=5$, and also

$$
\begin{aligned}
\lim f g x_{n} & =\lim f g\left(5+\frac{1}{n}\right)=\lim f\left(5+\frac{1}{n}\right) \\
& =\lim \left(\frac{5+1 / n+5}{2}\right)=5=g(5), \\
\lim g f x_{n} & =\lim g f\left(5+\frac{1}{n}\right)=\lim g\left(\frac{5+1 / n+5}{2}\right) \\
& =\lim g\left(5+\frac{1}{2 n}\right)=5 \neq f(5) .
\end{aligned}
$$

Therefore maps $f$ and $g$ are weak semicompatible.

It can easily be shown that maps $f$ and $g$ satisfy conditional commutativity at $x=1$. Moreover if we take sequence $x_{n}=5+1 / n, \lim f x_{n}=\lim g x_{n}=5$. 
Also, $\lim f f x_{n}=\lim f f(5+1 / n)=\lim f(5+1 / 2 n)=5=$ $g(5)$ and $\lim f g x_{n}=\lim f g(5+1 / n)=\lim f(5+1 / n)=5=$ $g(5)$. Hence maps $f$ and $g$ are $f$-compatible of type $(E)$. The mappings $f$ and $g$ are $R$-weakly commuting of type $A_{f}$; that is,

$$
d(f g x, g g x) \leq R d(f x, g x) \quad \text { with } R=1 \forall x \in X .
$$

Moreover the pair $(f, g)$ satisfies equally the condition of (b) if $x, y=1$. Again the pair $(f, g)$ satisfies equally the condition of (b) if $x, y \in(1,5)$ and $x, y=5$ for $a=2, b=-3$, and $c=3$.

Now finally if $f x=(x+5) / 2$ and $g x=x$ for $x, y>5$, the left hand side of given inequality yields $d(f x, f y)=\mid f x-$ $f y \mid=(x-y) / 2$ and right hand side gives

$$
\begin{aligned}
& a d(g x, g y)+b d(f x, g x)+c d(f y, g y) \\
2|x-y|-6\left|\frac{x+5}{2}-x\right|+6\left|\frac{y+5}{2}-y\right| & \\
\Longrightarrow & 2(x-y)-6\left(x-\frac{x+5}{2}\right)+6\left(y-\frac{y+5}{2}\right) \\
= & -(x-y) .
\end{aligned}
$$

Therefore the pair $(f, g)$ is satisfied greater than the condition of (b) when $a=2, b=-6$, and $c=6$, and 1 is a common fixed points of $f$ and $g$.

Corollary 17. Let $X$ be a set and let $d$ be the symmetric on $X$. Let maps $f$ and $g$ satisfy all the conditions of Theorem 15. Since either $f$ and $g$ are $f$-compatible of type (E) or $f$ and $g$ are $g$ compatible of type (E), then by Lemma 14 the pair $(f, g)$ will be owc and therefore the conclusion of Theorem 15 follows from Theorem 9 .

Theorem 18. Let $f$ and $g$ be weak semicompatible and selfmappings of a metric space $(X, d)$ such that

(a) $f(X) \subseteq g(X)$;

(b) $d(f x, f y) \geq a d(g x, g y)+b d(f x, g x)+c d(f y, g y)$;

$c>1$ and $a, b \in R$ such that $a+c>0 \& a+b+c>1$;

(c) $f$ and $g$ satisfying E.A. property.

If $f$ is $g$-absorbing or $g$ is $f$-absorbing, then $f$ and $g$ have a common fixed point in $X$.

Proof. Since $f$ and $g$ satisfy E.A. property, then there exists a sequence $\left\{x_{n}\right\}$ in $X$ such that $\lim f x_{n}=\lim g x_{n}=t$ for some $t$ in $X$.

Case 1 (suppose that $g$ is $f$-absorbing). Since $f$ and $g$ are weak semicompatible, this yields either $\lim f g x_{n}=g t$ or $\lim g f x_{n}=f t$.

First we take $\lim g f x_{n}=f t$.

Since $f(X) \subseteq g(X)$, for each sequence, $\left\{x_{n}\right\}$ there exists a sequence $\left\{y_{n}\right\}$ in $X$ such that $\lim f x_{n}=\lim g y_{n}$. Therefore, $\lim f x_{n}=\lim g y_{n}=t$ and $\lim g x_{n}=t$. By using (b), $d\left(f x_{n}, f y_{n}\right) \geq a d\left(g x_{n}, g y_{n}\right)+b d\left(f x_{n}, g x_{n}\right)+c d\left(f y_{n}, g y_{n}\right)$. Limiting $n \rightarrow \infty$ with $c>1$ yields

$$
\begin{array}{cc}
\lim f y_{n}=t, \quad \text { and so } & \lim f x_{n}=\lim g y_{n}=t, \\
\lim g x_{n}=t, & \lim f y_{n}=t .
\end{array}
$$

Since $g$ is $f$-absorbing, this yields $d\left(f y_{n}, f g y_{n}\right) \leq$ $R d\left(f y_{n}, g y_{n}\right)$. Limiting $n \rightarrow \infty$ yields $\lim f g y_{n}=t$. By (32) it is easy to see that $\lim f f x_{n}=t$. Now by (b), $d\left(f x_{n}, f f x_{n}\right) \geq$ $a d\left(g x_{n}, g f x_{n}\right)+b d\left(f x_{n}, g x_{n}\right)+c d\left(f f x_{n}, g f x_{n}\right)$. Limiting $n \rightarrow \infty$ yields $d(t, t) \geq a d(t, f t)+b d(t, t)+c d(t, f t)$. Since $a+c>0$, it gives $f t=t$. Since $\lim f f x_{n}=t$, therefore $\lim f f x_{n}=f t$.

Since $f(X) \subseteq g(X)$, then there exists a point $u$ in $X$ such that $f t=g u$. Now by (b), $d\left(f f x_{n}, f u\right) \geq a d\left(g f x_{n}, g u\right)+$ $b d\left(f f x_{n}, g f x_{n}\right)+c d(f u, g u)$. Limiting $n \rightarrow \infty$ yields

$$
\begin{aligned}
& d(f t, f u) \geq a d(f t, g u)+b d(f t, f t)+c d(f u, g u), \\
& d(g u, f u) \geq a d(g u, g u)+c d(f u, g u) .
\end{aligned}
$$

Since $c>1$, this yields $f u=g u$. Since $g$ is $f$-absorbing, this yields $d(f u, f g u) \leq R d(f u, g u)$ implying $f g u=f u$ or $f f u=f u$. Again by (b), we have

$$
\begin{gathered}
d(f u, f f u) \geq a d(g u, g f u)+b d(f u, g u)+c d(f f u, g f u), \\
d(f u, f u) \geq a d(f u, g f u)+b d(f u, f u)+c d(f u, g f u) .
\end{gathered}
$$

Since $a+c>0$, this yields $g f u=f u$ or $g f u=f f u=f u$. Hence $f u$ is common fixed point of $f$ and $g$.

Now we take $\lim f g x_{n}=g t$.

Since $g$ is $f$-absorbing, this yields $d\left(f x_{n}, f g x_{n}\right) \leq$ $R d\left(f x_{n}, g x_{n}\right)$. Limiting $n \rightarrow \infty$ yields $d(t, g t) \leq R d(t, t)$, which implies $g t=t$. Now by (b), $d\left(f x_{n}, f t\right) \geq a d\left(g x_{n}, g t\right)+$ $b d\left(f x_{n}, g x_{n}\right)+c d(f t, g t)$. Limiting $n \rightarrow \infty$ yields $d(t, f t) \geq$ $a d(t, t)+b d(t, t)+c d(f t, t)$. Since $c>1$, this yields $f t=t$. Hence $f t=g t=t$ or $t$ is common fixed point of $f$ and $g$.

Case 2 (suppose $f$ is $g$-absorbing). By virtue of (32) it is easy to see that $\lim f y_{n}=\lim g y_{n}=t$.

Since $f$ and $g$ are weak semicompatible mappings, this yields $\lim f g y_{n}=g t \& \lim g f y_{n}=f t$.

First we take $\lim g f y_{n}=f t$.

Since $f$ is $g$-absorbing, this yields $d\left(g y_{n}, g f y_{n}\right) \leq$ $R d\left(f y_{n}, g y_{n}\right)$. Limiting $n \rightarrow \infty$ yields $\lim g f y_{n}=t$. This implies $f t=t$. Now by (b), $d\left(f y_{n}, f f y_{n}\right) \geq a d\left(g y_{n}, g f y_{n}\right)+$ $b d\left(f y_{n}, g y_{n}\right)+c d\left(f f y_{n}, g f y_{n}\right)$. Limiting $n \rightarrow \infty$ yields $d\left(t, f f y_{n}\right) \geq a d(t, t)+b d(t, t)+c d\left(f f y_{n}, t\right)$. Since $c>1$, this yields $\lim f f y_{n}=t$ or $\lim f f y_{n}=f t$.

Since $f(X) \subseteq g(X)$, then there exists a point $u$ in $X$ such that $f t=g u$. Now by (b), $d\left(f f y_{n}, f u\right) \geq a d\left(g f y_{n}, g u\right)+$ $b d\left(f f y_{n}, g f y_{n}\right)+c d(f u, g u)$. Limiting $n \rightarrow \infty$ yields

$$
\begin{aligned}
& d(f t, f u) \geq a d(f t, f t)+b d(f t, f t)+c d(f u, g u), \\
& d(g u, f u) \geq c d(f u, g u) .
\end{aligned}
$$


Since $c>1$, this yields $f u=g u$. Since $f$ is $g$-absorbing, this yields $d(g u, g f u) \leq R d(f u, g u)$ implying $g f u=f u$. Again by (b), we have

$$
\begin{aligned}
& d(f u, f g u) \geq a d(g u, g g u)+b d(f u, g u)+c d(f g u, g g u) \\
& d(f u, f f u) \geq a d(f u, f u)+b d(f u, f u)+c d(f f u, f u)
\end{aligned}
$$

Since $c>1$, this yields $f f u=f u$ or $g f u=f f u=f u$. Hence $f u$ is common fixed point of $f$ and $g$.

Now we take $\lim f g y_{n}=g t$.

Since $f$ is $g$-absorbing, this yields $d\left(g x_{n}, g f x_{n}\right) \leq$ $R d\left(g x_{n}, f x_{n}\right)$ and $d\left(g y_{n}, g f y_{n}\right) \leq R d\left(g y_{n}, f y_{n}\right)$. Limiting $n \rightarrow \infty$ yields $\lim g f x_{n}=t \& \lim g f y_{n}=t$. By virtue of (32) $\lim g f x_{n}=\lim g g y_{n}=t$.

Now by (b), $d\left(f y_{n}, f g y_{n}\right) \geq a d\left(g y_{n}, g f y_{n}\right)+b d\left(f y_{n}, g y_{n}\right)+$ $c d\left(f g y_{n}, g g y_{n}\right)$. Limiting $n \rightarrow \infty$ yields $d(t, g t) \geq a d(t, t)+$ $b d(t, t)+c d(g t, t)$. Since $c>1$, this yields $g t=t$.

Now by (b), $d\left(f y_{n}, f t\right) \geq a d\left(g y_{n}, g t\right)+b d\left(f y_{n}, g y_{n}\right)+$ $c d(f t, g t)$. Limiting $n \rightarrow \infty$ yields $d(t, f t) \geq a d(t, t)+$ $b d(t, t)+c d(f t, t)$. Since $c>1$, this yields $f t=t$. Hence $f t=g t=t$ or $t$ is common fixed point of $f$ and $g$.

Example 19. Let $x, y \in X(x \geq y)$ and $X=[1,10]$ and $d$ be the usual metric on $X$. Define $f, g: X \rightarrow X$ as follows:

$$
\begin{array}{ll}
f x=1 & \text { if } x=1, \\
f x=5 & \text { if } x>5, \\
f x=6 & \text { if } 1<x \leq 5, \\
g x=1 & \text { if } x=1, \\
g x=x & \text { if } x>5, \\
g x=5 & \text { if } 1<x \leq 5 .
\end{array}
$$

When we take constant sequence $\left\{x_{n}\right\}=1$, then $\lim f x_{n}=$ $\lim g x_{n}=1, \lim f g x_{n}=1=g(1)$, and $\lim g f x_{n}=1=f(1)$. But if we take sequence $x_{n}=5+1 / n, \lim f x_{n}=\lim g x_{n}=5$, and also

$$
\begin{gathered}
\lim f g x_{n}=\lim f g\left(5+\frac{1}{n}\right)=\lim f\left(5+\frac{1}{n}\right)=5=g(5), \\
\lim g f x_{n}=\lim g f\left(5+\frac{1}{n}\right)=\lim g(5)=5 \neq f(5)
\end{gathered}
$$

Therefore maps $f$ and $g$ are weak semicompatible.

It can be easily seen that maps $f$ and $g$ satisfy E.A. property. Moreover map $g$ is $f$-absorbing at $x=1$. Map $g$ is $f$-absorbing at $1<x \leq 5$ for $R>0$ and map $g$ is $f$ absorbing at $x>5$ for $R>0$. Moreover the pair $(f, g)$ satisfies equally the condition of (b) if $x, y=1$ and the pair $(f, g)$ satisfies equally the condition of (b) for $x, y \in(1,5]$ when $a=2, b=-3$, and $c=3$. Now finally if $f x=5$ and $g x=x$ for $x, y>5$, the left hand side of the given inequality yields $d(f x, f y)=|f x-f y|=0$ and right hand side gives

$$
\begin{aligned}
& \text { ad }(g x, g y)+b d(f x, g x)+c d(f y, g y), \\
& 2|x-y|-6|5-x|+6|5-y| \\
& \quad \Longrightarrow 2(x-y)-6(x-5)+6(y-5) \\
& =4(-x+y) \text { (negative or zero) }
\end{aligned}
$$

Therefore the pair $(f, g)$ is satisfied greater than or is equal to the condition of (b) when $a=2, b=-6$, and $c=6$, and 1 is a common fixed points of $f$ and $g$.

Theorem 20. Let $f$ and $g$ be weak semicompatible and selfmappings of a metric space $(X, d)$ such that

(a) $f(X) \subseteq g(X)$;

(b) $d(f x, f y) \geq a d(g x, g y)+b d(f x, g x)+c d(f y, g y)$; $a, c>1(a+c>0)$ and $b \in R$ such that $a+b+c>1$;

(c) $f$ and $g$ satisfying E.A. property.

If $f$ and $g$ are $f$-compatible or $f$ and $g$ are $g$-compatible, then $f$ and $g$ have a common fixed point in $X$.

Proof. Since $f$ and $g$ satisfy E.A. property, then there exists a sequence $\left\{x_{n}\right\}$ in $X$ such that $\lim f x_{n}=\lim g x_{n}=t$ for some $t$ in $X$.

Case 1 (suppose $f$ and $g$ are $f$-compatible). Since $f$ and $g$ are weak semicompatible, this yields either $\lim f g x_{n}=g t$ or $\lim g f x_{n}=f t$.

First we take $\lim g f x_{n}=f t$.

Since $f(X) \subseteq g(X)$, for each sequence, $\left\{x_{n}\right\}$ there exists a sequence $\left\{y_{n}\right\}$ in $X$ such that $\lim f x_{n}=\lim g y_{n}$. Therefore, $\lim f x_{n}=\lim g y_{n}=t$, and $\lim g x_{n}=t$. By using (b), $d\left(f x_{n}, f y_{n}\right) \geq \operatorname{ad}\left(g x_{n}, g y_{n}\right)+b d\left(f x_{n}, g x_{n}\right)+c d\left(f y_{n}, g y_{n}\right)$. Limiting $n \rightarrow \infty$ with $c>1$ yields $\lim f y_{n}=t$, so

$$
\lim f x_{n}=\lim g y_{n}=t, \quad \lim g x_{n}=t, \quad \lim f y_{n}=t .
$$

Since $f$ and $g$ are $f$-compatible, this yields $\lim d\left(f g y_{n}\right.$, $\left.g g y_{n}\right)=0$ or $\lim f g y_{n}=\lim g g y_{n}$. By virtue of (40) $\lim g f x_{n}=\lim g g y_{n}=f t$. Therefore, $\lim f g y_{n}=f t$. Now by (b), $d\left(f g y_{n}, f t\right) \geq a d\left(g g y_{n}, g t\right)+b d\left(f g y_{n}, g g y_{n}\right)+c d(f t, g t)$. Limiting $n \rightarrow \infty$ yields $d(f t, f t) \geq a d(f t, g t)+b d(f t, f t)+$ $c d(f t, g t)$. Since $a+c>0$, this yields $f t=g t$.

Since $f$-compatibility of $f$ and $g$ implies commutativity at coincidence point, this gives $f g t=g f t$ or $f g t=g f t=$ $f f t=g g t$. Now by (b), we get

$$
\begin{aligned}
& d(f f t, f t) \geq a d(g f t, g t)+b d(f f t, g f t)+c d(f t, g t), \\
& d(f f t, f t) \geq a d(f f t, f t) .
\end{aligned}
$$

Since $a>1$, this implies $f f t=f t$ or $f f t=g f t=f t$. Hence $f t$ is common fixed point of $f$ and $g$.

Now we take $\lim f g x_{n}=g t$. 
Since $f$ and $g$ are $f$-compatible, this yields $\lim d\left(f g x_{n}, g g x_{n}\right)=0$ or $\lim f g x_{n}=\lim g g x_{n}$. Therefore, $\lim g g x_{n}=g t$. Now by (b), $d\left(f g x_{n}, f t\right) \geq$ $a d\left(g g x_{n}, g t\right)+b d\left(f g x_{n}, g g x_{n}\right)+c d(f t, g t)$. Limiting $n \rightarrow \infty$ yields $d(g t, f t) \geq a d(g t, g t)+b d(g t, g t)+c d(f t, g t)$. Since $c>1$, this implies $f t=g t$.

Since $f$-compatibility of $f$ and $g$ implies commutativity at coincidence point, this gives $f g t=g f t$ or $f g t=g f t=$ $f f t=g g t$. Now by $(\mathrm{b})$, we get

$$
\begin{aligned}
& d(f f t, f t) \geq a d(g f t, g t)+b d(f f t, g f t)+c d(f t, g t), \\
& d(f f t, f t) \geq a d(f f t, f t) .
\end{aligned}
$$

Since $a>1$, this implies $f f t=f t$ or $f f t=g f t=f t$. Hence $f t$ is common fixed point of $f$ and $g$.

Case 2 (suppose $f$ and $g$ are $g$-compatible). Since $f$ and $g$ are weak semicompatible, this yields either $\lim f g x_{n}=g t$ or $\lim g f x_{n}=f t$.

First we take $\lim g f x_{n}=f t$.

Since $f$ and $g$ are $g$-compatible, this yields $\lim d\left(g f x_{n}, f f x_{n}\right)=0$ or $\lim g f x_{n}=\lim f f x_{n}$. Therefore $\lim f f x_{n}=f t$. Now by (b), $d\left(f f x_{n}, f t\right) \geq$ $a d\left(g f x_{n}, g t\right)+b d\left(f f x_{n}, g f x_{n}\right)+c d(f t, g t)$. Limiting $n \rightarrow \infty$ yields $d(f t, f t) \geq a d(f t, g t)+b d(f t, f t)+c d(f t, g t)$. Since $a+c>0$, this implies $f t=g t$.

Since $g$-compatibility of $f$ and $g$ implies commutativity at coincidence point, this gives $f g t=g f t$ or $f g t=g f t=$ $f f t=g g t$. Now by (b), we have

$$
\begin{aligned}
& d(f f t, f t) \geq a d(g f t, g t)+b d(f f t, g f t)+c d(f t, g t), \\
& d(f f t, f t) \geq a d(f f t, f t) .
\end{aligned}
$$

Since $a>1$, this implies $f f t=f t$ or $f f t=g f t=f t$. Hence $f t$ is common fixed point of $f$ and $g$.

Now we take $\lim f g x_{n}=g t$.

Since $f$ and $g$ are $g$-compatible, this yields

$$
\lim d\left(g f x_{n}, f f x_{n}\right)=0 \quad \text { or } \quad \lim g f x_{n}=\lim f f x_{n} .
$$

Since $f$ and $g$ satisfy E.A. property, this yields with (44) $\lim g g x_{n}=\lim f g x_{n}=g t$. Now by (b), $d\left(f g x_{n}, f t\right) \geq$ $a d\left(g g x_{n}, g t\right)+b d\left(f g x_{n}, g g x_{n}\right)+c d(f t, g t)$. Limiting $n \rightarrow \infty$ yields $d(g t, f t) \geq a d(g t, g t)+b d(g t, g t)+c d(f t, g t)$. Since $c>1$, this implies $f t=g t$.

Since $g$-compatibility of $f$ and $g$ implies commutativity at coincidence point, this gives $f g t=g f t$ or $f g t=g f t=$ $f f t=g g t$. Now by (b), we have

$$
\begin{aligned}
& d(f f t, f t) \geq a d(g f t, g t)+b d(f f t, g f t)+c d(f t, g t), \\
& d(f f t, f t) \geq a d(f f t, f t) .
\end{aligned}
$$

Since $a>1$, this implies $f f t=f t$ or $f f t=g f t=f t$. Hence $f t$ is common fixed point of $f$ and $g$.
Example 21. Let $x, y \in X(x \geq y)$ and $X=[1,25]$ and $d$ be the usual metric on $X$. Define $f, g: X \rightarrow X$ as follows:

$$
\begin{aligned}
& f x=1 \quad \text { if } x=1, x \geq 5, \\
& f x=5 \quad \text { if } 1<x<5, \\
& g x=1 \quad \text { if } x=1, \\
& g x=x+4 \quad \text { if } 1<x<5, \\
& g x=\frac{x}{5} \quad \text { if } x \geq 5,
\end{aligned}
$$

if we take constant sequence $x_{n}=1, \lim f x_{n}=\lim g x_{n}=1$, $\lim f g x_{n}=\lim f(1)=1=g(1)$, and $\lim g f x_{n}=\lim g(1)=$ $1=f(1)$, but when we take sequence $x_{n}=5+1 / n$, then $\lim f x_{n}=1$ and $\lim g x_{n}=\lim ((5+1 / n) / 5)=1$ or $\lim f x_{n}=$ $\lim g x_{n}=1$. Moreover $\lim f g x_{n}=\lim f((5+1 / n) / 5)=$ $\lim f(1+1 / 5 n)=5 \neq g(1)$ and $\lim g f x_{n}=\lim g(1)=1=$ $f(1)$.

Therefore $f$ and $g$ are weak semicompatible. It can be easily shown that mappings $f$ and $g$ satisfy E.A. property. When we take sequence $x_{n}=1$, it is easy to show that maps $f$ and $g$ are $f$-compatible. When we take sequence $x_{n}=5+1 / n$, then $\lim f x_{n}=\lim f(5+1 / n)=1$ and $\lim g x_{n}=\lim g(5+1 / n)=1$; therefore $\lim f x_{n}=\lim g x_{n}=1$.

Now $\lim f g x_{n}=\lim f g(5+1 / n)=5$ and $\lim g g x_{n}=$ $\lim g g(5+1 / n)=\lim g(1+1 / 5 n)=5$. Thus $\lim f g x_{n}=$ $\lim g g x_{n}$. Therefore maps $f$ and $g$ are $f$-compatible. Again if we take sequence $x_{n}=1+1 / n$, then $\lim f x_{n}=\lim f(1+1 / n)=$ 5 and $\lim g x_{n}=\lim g(1+1 / n)=5$; thus $\lim f x_{n}=\lim g x_{n}=$ 1. Now $\lim f g x_{n}=\lim f g(1+1 / n)=\lim f(5+1 / n)=1$ and $\lim g g x_{n}=g g(1+1 / n)=g(5+1 / n)=1$. Thus $\lim f g x_{n}=$ $\lim g g x_{n}$. Therefore maps $f$ and $g$ are $f$-compatible.

Moreover the pair $(f, g)$ is satisfying equally the condition of (b) if $x, y=1$ and if we take $f x=1$ and $g x=x / 5$, for $x, y \geq 5$, left hand side gives $d(f x, f y)=|f x-f y|=0$ and right hand side gives

$$
\begin{aligned}
& a d(g x, g y)+b d(f x, g x)+c d(f y, g y), \\
& 4\left|\frac{x}{5}-\frac{y}{5}\right|-4\left|1-\frac{x}{5}\right|+3\left|1-\frac{y}{5}\right| \\
& \quad \Longrightarrow 4\left(\frac{x}{5}-\frac{y}{5}\right)-4\left(\frac{x}{5}-1\right)+3\left(\frac{y}{5}-1\right) \\
& \quad=1-\frac{y}{5} \text { (negative or zero). }
\end{aligned}
$$

Therefore the pair $(f, g)$ is satisfied greater than or is equal to the condition of (b) when $a=4, b=-4$, and $c=3$.

Now finally if $f x=5$ and $g x=x+4$ for $1<x<5$. The left hand side of given inequality yields $d(f x, f y)=|f x-f y|=0$ and right hand side gives

$$
\begin{aligned}
& \quad a d(g x, g y)+b d(f x, g x)+c d(f y, g y), \\
& 4|(x-4)-(y-4)|-4|5-(x-4)|+3|5-(y-4)| \\
& \quad \Longrightarrow 4(x-y)-4(x-1)+3(y-1) \\
& =1-y \text { (negative). }
\end{aligned}
$$


Therefore the pair $(f, g)$ is satisfied greater than the condition of (b) when $a=4, b=-4$, and $c=3$, and 1 is a common fixed points of $f$ and $g$.

Corollary 22. Let $X$ be a set and let $d$ be the symmetric on $X$. Let maps $f$ and $g$ satisfy all the conditions of Theorem 20. Since $f$ and $g$ are either $f$-compatible or $g$-compatible, then, by Lemma 11, pair $(f, g)$ will be owc and therefore the conclusion of Theorem 20 follows from Theorem 9.

\section{Conflict of Interests}

The authors declare that there is no conflict of interests regarding the publication of this paper.

\section{References}

[1] Y. J. Cho, B. K. Sharma, and D. R. Sahu, "Semi compatibility and fixed points," Mathematica Japonica, vol. 42, no. 1, pp. 9198, 1995.

[2] B. Singh and S. Jain, "Semi compatibility, compatibility and fixed point theorems in fuzzy metric spaces," Journal of the Chungcheong Mathematical Society, vol. 18, pp. 1-22, 2005.

[3] S. Sessa, "On a weak commutativity condition of mappings in fixed point considerations," Publications de l'Institut Mathématique, vol. 32, pp. 149-153, 1982.

[4] G. Jungck, "Compatible mappings and common fixed points," International Journal of Mathematics and Mathematical Sciences, vol. 9, no. 4, pp. 771-779, 1986.

[5] G. Jungck, P. P. Murthy, and Y. J. Cho, "Compatible mappings of type (A) and common fixed points," Mathematica Japonicae, vol. 38, no. 2, pp. 381-390, 1993.

[6] H. K. Pathak and M. S. Khan, "A comparision of various types of compatible maps and common fixed points," Indian Journal of Pure and Applied Mathematics, vol. 28, no. 4, pp. 477-485, 1997.

[7] S. L. Singh and A. Tomar, "Weaker forms of commuting maps and existence of fixed points," Journal of the Korean Society of Mathematical Education, vol. 3, pp. 145-161, 2003.

[8] R. P. Pant, "Common fixed points of noncommuting mappings," Journal of Mathematical Analysis and Applications, vol. 188, no. 2, pp. 436-440, 1994.

[9] H. K. Pathak, Y. J. Cho, and S. M. Kang, "Remarks of R-weakly commuting mappings and common fixed point theorems," Bulletin of the Korean Mathematical Society, vol. 34, pp. 247-257, 1997.

[10] D. Gopal, A. S. Ranadive, and R. P. Pant, "Common fixed points of absorbing maps," Bulletin of Marathwada Mathematical Society, vol. 9, pp. 43-48, 2008.

[11] M. Aamri and D. El Moutawakil, "Some new common fixed point theorems under strict contractive conditions," Journal of Mathematical Analysis and Applications, vol. 270, no. 1, pp. 181188, 2002.

[12] M. R. Singh and Y. M. Singh, "On various types of compatible maps and common fixed point theorems for non-continuous maps," Hacettepe Journal of Mathematics and Statistics, vol. 40, no. 4, pp. 503-513, 2011.

[13] R. P. Pant, R. K. Bisht, and D. Arora, "Weak reciprocal continuity and fixed point theorems," Annali dell'Universita di Ferrara, vol. 57, no. 1, pp. 181-190, 2011.
[14] A. S. Saluja, M. K. Jain, and P. K. Jhade, "Weak semi compatibility and fixed point theorems," Bulletin of International Mathematical Virtual Institute, vol. 2, pp. 205-217, 2012.

[15] G. Jungck and B. E. Rhoades, "Fixed point theorems for occasionally weakly compatible mappings," Fixed Point Theory, vol. 7, no. 2, pp. 287-296, 2006.

[16] M. A. Al-Thagafi and N. Shahzad, "Generalized I-nonexpansive selfmaps and invariant approximations," Acta Mathematica Sinica, vol. 24, no. 5, pp. 867-876, 2008.

[17] V. Pant and R. P. Pant, "Common fixed points of conditionally commuting maps," Fixed Point Theory, vol. 11, no. 1, pp. 113-118, 2010.

[18] H. Bouhadjera, "On common fixed point theorems for three and four self mappings satisfying contractive conditions," Acta Universitatis Palackianae Olomucensis, vol. 49, no. 1, pp. 25-31, 2010. 


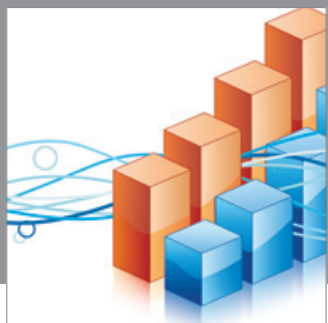

Advances in

Operations Research

mansans

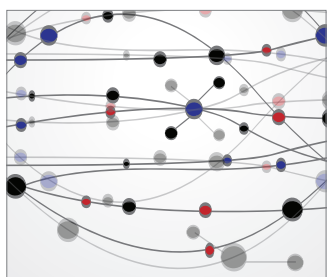

The Scientific World Journal
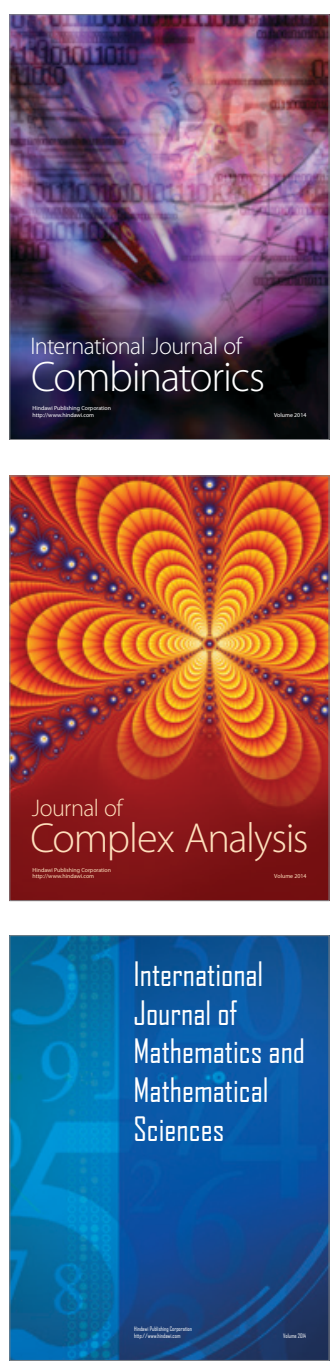
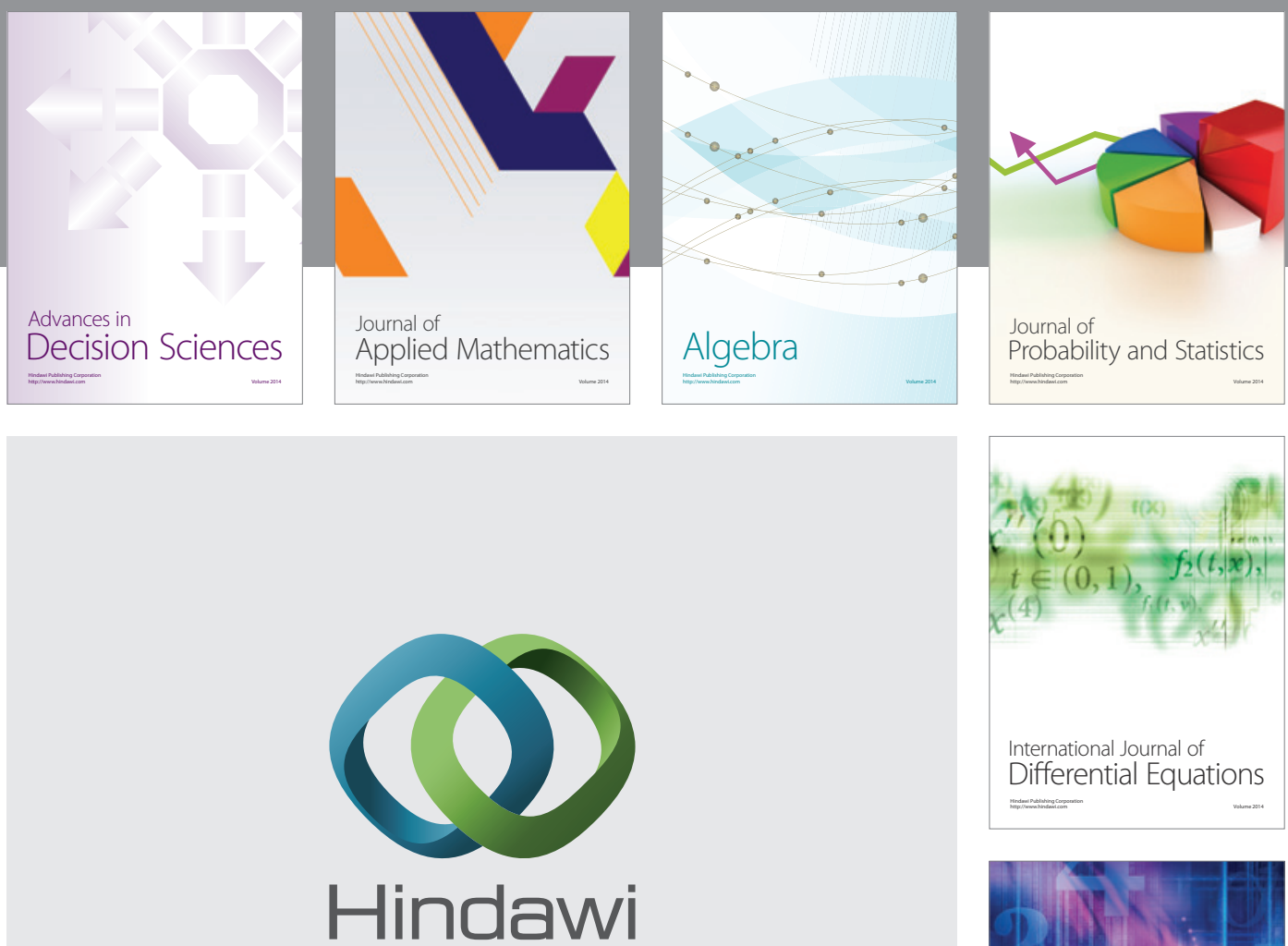

Submit your manuscripts at http://www.hindawi.com
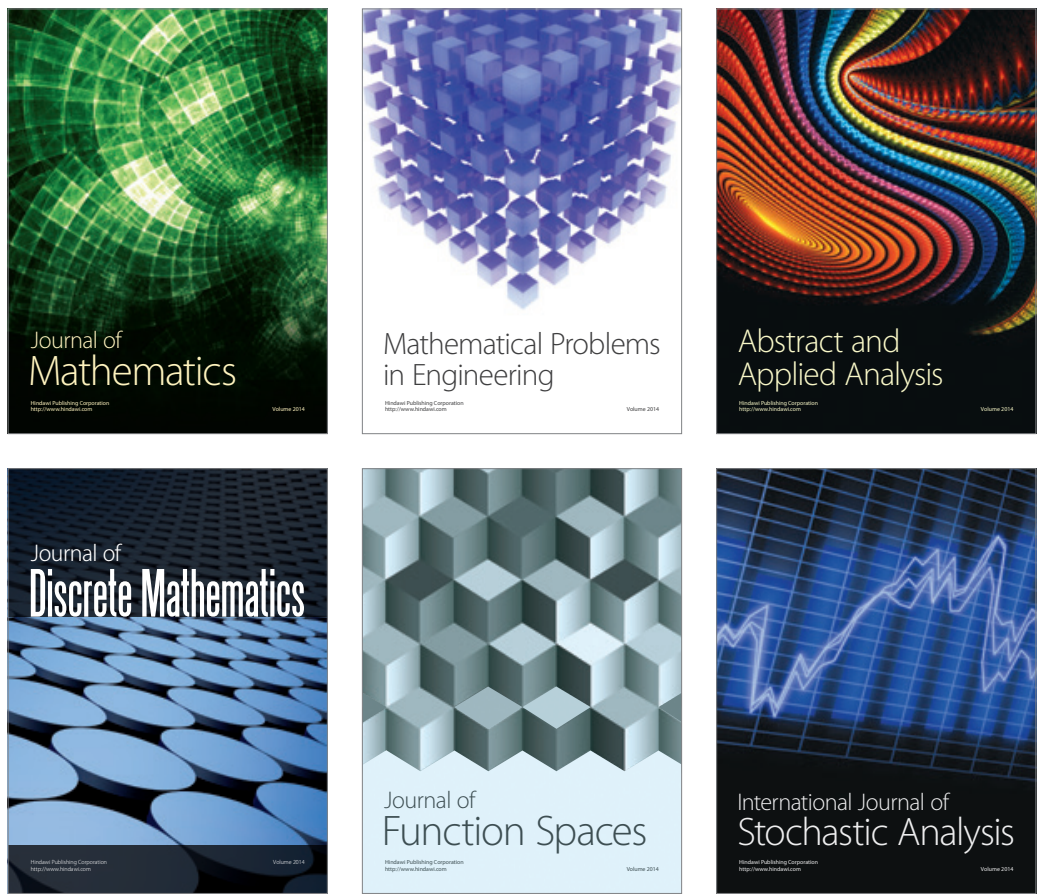

Journal of

Function Spaces

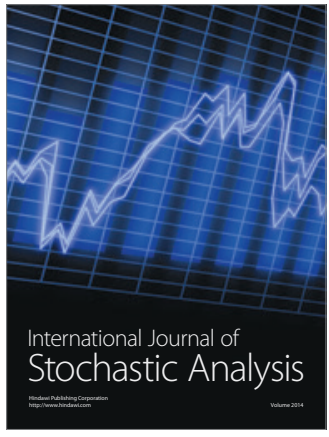

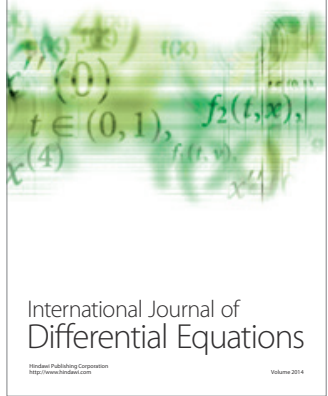
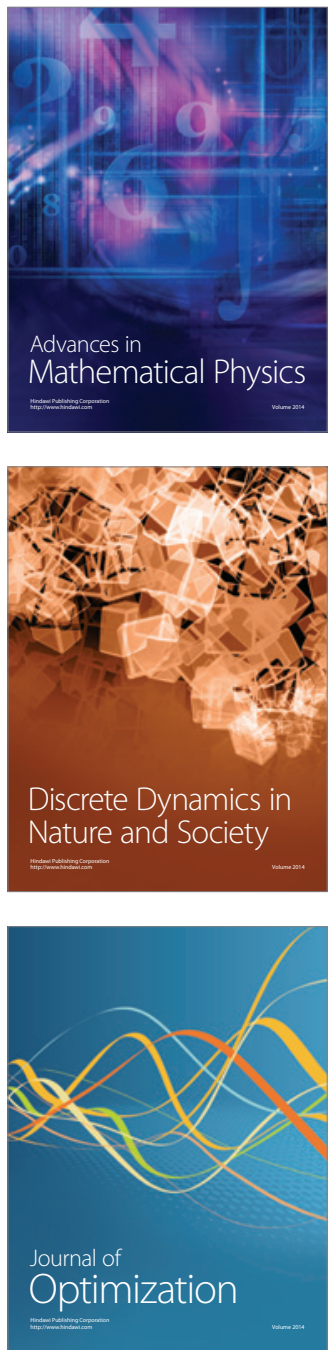